\title{
Le contexte : reconversion de la chimie vers la chimie verte
}

Hilaire BEWA

Virginie LE RAVALEC

\author{
ADEME, \\ Direction Productions et Energies \\ Durables, \\ Service Bioressources, \\ 20, avenue du Grésillé - 49004 Angers \\ cedex 01 \\ $<$ hilaire.bewa@ademe.fr>
}

\begin{abstract}
Chemical industry is a key sector on the world industrie with a twolrd urnover that reached 1871 billions of euros in 2009 and with an annual turnove in France of 77 billions in 2010. This industry, essentially based on the use of of fossil ressources (most of all oil), has been facing for several years significant challenges: scarcity of raw material, strengthening of the regulatory structure in the European Union (REACH), international competition from emerging countries. .. In this context, chemical industry is currently undergoing fundamental changes to a cleaner and smore ustainable chemistry. In France, a particular emphasis was placed on the vegetal-based chemistry which represents a significant potential for innovation and a substantial market. So, numerous initiatives have been taken in order to increase the competitiveness of this sector, integrating all players involved (biomass producers, agribusiness, chemists, distributors. .). The market of bio-sourced products derived from this chemistry is still weak, but has been recording continual growth for several years now.
\end{abstract}

Key words: green chemistry, bio-sourced products, France, context, initiatives, competitiveness, impediments

\section{L'industrie chimique et sa reconversion}

de l'industrie mondiale. Elle contribue au développement des autres activités manufacturières et participe à la croissance de l'économie. Le secteur de la chimie mondiale représente 1871 milliards d'euros en 2009, celui de I'Union européenne 494 milliards en 2010 avec une croissance annuelle estimée à $2 \%$ en 2011 par an. La France avec plus de 77 milliards d'euros en 2010 occupe le $5^{\mathrm{e}}$ rang mondial et le $2^{\mathrm{e}}$ rang européen. Cette industrie génère plus de 1200000 emplois à l'échelle européenne dont environ 180000 emplois directs en France. L'industrie chimique regroupe plusieurs activités, de nombreux savoir-faire et des marchés finaux très différents (agriculture, agro-alimentaire, pharmacie, automobile, construction...), le tout reposant sur une gamme de produits et de biens de consommation extrêmement hétérogènes issus majoritairement de matières premières fossiles et non renouvelables (pétrole, gaz).
L'industrie chimique française, comme l'ensemble de l'industrie chimique européenne, est confrontée, depuis plusieurs années, à d'importants défis, notamment des défis liés, d'une part, à la raréfaction des ressources pétrolières dont elle tire la majorité de ses produits et, d'autre part, au renforcement du cadre réglementaire (en particulier REACH), qui font de l'industrie chimique un des principaux secteurs concernés par les enjeux environnementaux. On peut citer également les défis concernant l'émergence d'une concurrence internationale forte de nouveaux acteurs de pays émergents (Chine et Inde principalement) qui accueillent de plus en plus de sites industriels pour les secteurs clients de la chimie comme l'électronique, les plastiques ou les textiles, ou enfin, des défis relatifs à la faiblesse d'innovation et $\mathrm{d}^{\prime}$ efforts en recherche et développement, I'une des conséquences étant la délo- calisation des industries traditionnelles. $C^{\prime}$ est dans ce contexte que s'est engagée, ces dernières années, en France, une réflexion approfondie sur le devenir de I'industrie chimique française et sa reconversion. Ainsi, en 2005, le groupe de réflexion stratégique publiait un rapport sur l'avenir de l'industrie chimique à I'horizon 2015 et proposait notamment la mise en place de filières stratégiques de progrès et I'orientation vers une chimie durable. En 2010, le PIPAME (Pôle interministériel de prospective et d'anticipation des mutations économiques), sous l'égide de la DGCIS (Direction générale de la compétitivité, de l'industrie et des services), a publié un rapport clé "Mutations économiques dans le domaine de la chimie » positionnant l'essor de la chimie du végétal comme apportant une contribution importante à la chimie durable, ce qui marque la mutation actuelle de la chimie traditionnelle vers une chimie durable et la volonté des pouvoirs publics d'accompagner cette transition. Dans cette optique, I'ADEME a publié en

Pour citer cet article : Bewa H, Le Ravalec V. Le contexte : reconversion de la chimie vers la chimie verte. OCL 2012 ; 19(1) : 1-5. doi : 10.1684/ ocl.2012.0424 
avril 2011, dans le cadre de l'Investissement d'Avenir, une feuille de route stratégique chimie du végétal qui vise à éclairer les choix pour accompagner la mutation des technologies traditionnelles de l'industrie chimique vers des procédés cherchant à incorporer une part de plus en plus importante de matières premières végétales (céréales, betteraves, ressources lignocellulosiques, algues...). Au total, les actions et les recommandations suggérées dans cette feuille de route devront permettre de développer sur le territoire national une filière chimie du végétal compétitive intégrant I'ensemble des acteurs (producteurs de biomasse, agro-industriels, chimistes, distributeurs...). Cette feuille de route stratégique a servi de socle pour l'élaboration, puis le lancement d'un appel à manifestation d'intérêt (AMI) sur la chimie du végétal, l'objectif étant " de contribuer à rendre opérationnelle et compétitive commercialement, à court et à plus long terme, la production de produits biosourcés ".

\section{La chimie du végétal}

On peut considérer que la chimie du végétal est un sous-ensemble de la chimie verte car elle répond au principe $\mathrm{n}^{\circ} 7$ qui préconise I'utilisation de ressources renouvelables à la place de ressources fossiles lorsque la technologie et les moyens financiers le permettent. Le concept de chimie du végétal ne renie pas les onze autres principes de la chimie verte mais se donne pour objectifs principaux: i) le remplacement de l'ensemble des fonctionnalités offertes actuellement par la pétrochimie, par des produits issus de matières premières végétales, soit en reproduisant des molécules existantes à partir de matière première renouvelable, soit en produisant des molécules biosourcées présentant des fonctionnalités (usages) similaires à celles des molécules d'origine pétrochimique (innovation de substitution), soit en élaborant des molécules dotées de nouvelles fonctionnalités permettant de valoriser les caractéristiques propres des matières premières végétales (innovation de rupture); ii) la réponse aux défis environnementaux actuels dans le cadre du développement durable : lutte contre l'effet de serre, réduction des pollutions (air, sol, eau), innocuité (cf. directive européenne REACH) et biodégradabilité des produits. La chimie du végétal se distingue de la chimie verte à la fois par la nature du carbone utilisé (carbone renouvelable) et par les procédés mis en œuvre : procédés physico-chimiques et thermiques classiques permettant de valoriser la plante entière par voie chimique, sucrochimie qui valorise les sucres contenus dans le végétal par voie biologique, oléochimie ou lipochimie qui vise à transformer les huiles contenues dans les plantes oléo-protéagineuses, enfin, de nouveaux procédés des biotechnologies blanches ou biotechnologies industrielles qui valorisent des biomasses complexes par voie biologique. Ces biotechnologies industrielles devraient s'intégrer à plus ou moins long terme au sein de bioraffineries. II faut noter que le développement des biotechnologies donne de nouvelles perspectives à la sucrochimie grâce à la conception et à la mise en œuvre de micoorganismes de plus en plus performants. En effet, les biotechnologies industrielles connaissent un essor remarquable et représentent un facteur clé dans le développement de la filière chimie du végétal dans son ensemble. Elles suscitent beaucoup d'espoir pour valoriser la lignocellulose. Les biotechnologies industrielles ont été identifiées par la plateforme technologique européenne SusChem comme présentant un potentiel fort à la fois pour l'économie et la durabilité de la chimie, notamment en favorisant la conversion des ressources renouvelables en un nombre d'étapes limité et en évitant la production de sous-produits indésirables. Le potentiel des biotechnologies industrielles sera fortement conforté par le développement de la biologie de synthèse, qui vise à transformer, par ingénierie métabolique, les cellules microbiennes (bactéries, levures, champignons, micro-algues) en véritables usines cellulaires performantes pour la production de composés d'intérêt ou intermédiaires chimiques biosourcés. On peut déjà noter que le nombre de projets industriels concrets se multiplie mais reste encore limité, particulièrement en ce qui concerne la production d'intermédiaires chimiques car les changements d'échelle sont longs et difficiles, les investissements industriels importants et des risques demeurent sur les marchés.

\section{Le marché des produits issus de la chimie du végétal ou produits biosourcés}

Parmi les principaux produits biosourcés disponibles sur le marché depuis plusieurs années, on peut citer :

- les intermédiaires chimiques biosourcés qui peuvent être utilisés directement (éthanol) ou rentrer dans la formulation d'autres produits (acides carboxyliques (exemples: acide succinique, acétique, lactique), épichlorhydrine, acroléine, 1,3-propanediol, isosorbide, acides gras, polyols, etc., et dont le potentiel est considérable. Le marché de ces intermédiaires chimiques biosourcés est en nette progression dans le monde (tableau 1);

- matériaux biosourcés (polymères, plastiques végétaux et composites);

- produits fonctionnalisés, destinés à être soit formulés, soit directement mis sur le marché : solvants, tensioactifs, lubrifiants, plastifiants, encres, colles et peintures, etc.

Les domaines d'application sont vastes et divers: emballage, films agricoles et industriels, transport (carrosserie, joints, pièces de moteur, tuyaux...), bâtiment (isolation, portes, cloisons...), équipements électriques/électroniques, cosmétique (parfums, maquillage...), pharmaceutique, alimentation (arômes, additifs), détergence, dégraissage, forage, hydraulique, traitement du bois, revêtements.

D'après différentes sources, le marché de la chimie du végétal atteindra de I'ordre de 300 milliards d'euros à I'horizon 2020, avec une croissance de 10 à $15 \%$ par an. Le développement des produits biosourcés a pris de l'avance aux États-Unis comparativement à I'Union européenne car il a été largement soutenu par les autorités, notamment au travers des programmes du "USDA's Biobase Procurement Model " depuis 2002 et plus récemment du programme "USDA's BioPreferred program " grâce auquel plus de 1000 produits biosourcés se sont ajoutés aux 3500 produits biosourcés déjà éligibles pour les achats publics au niveau fédéral. Grâce à ces financements publics et à ces politiques publiques incitatives, les États-Unis sont aujourd'hui leader mondial sur les produits biosourcés à travers les applica- 
Tableau 1. Production mondiale d'intermédiaires chimiques biosourcés passée, actuelle et prospectives

\begin{tabular}{|c|c|c|c|c|c|c|}
\hline & & 2005 & 2010 & $\begin{array}{l}2020 \\
\text { sans } \\
\text { contraintes }\end{array}$ & $\begin{array}{l}2020 \\
\text { avec } \\
\text { contraintes }\end{array}$ & $\begin{array}{l}\text { Croissance } \\
2020 \text { vs. } \\
2005^{*}\end{array}$ \\
\hline \multicolumn{2}{|l|}{ Mondial } & $\begin{array}{l}56 \text { milliards } € \\
(7 \%)\end{array}$ & $\begin{array}{l}91 \text { milliards } € \\
(10 \%)\end{array}$ & 182 milliards $€$ & & $\times 3,2$ \\
\hline \multirow{9}{*}{$\begin{array}{l}\text { Intermédiaires } \\
\text { chimiques niveau } \\
\text { mondial }\end{array}$} & Acide succinique & & $\begin{array}{l}2,5 \mathrm{kt}(8,3 \%) \\
6250 \mathrm{k} €\end{array}$ & $\begin{array}{l}1000 \mathrm{kt} \\
2500000 \mathrm{k} €\end{array}$ & $\begin{array}{l}2000 \mathrm{kt} \\
5000000 \mathrm{k} €\end{array}$ & $\times 200$ \\
\hline & 1,3-propanediol & & $\begin{array}{l}45 \mathrm{kt}(53 \%) \\
85500 \mathrm{k} €\end{array}$ & $\begin{array}{l}1400 \mathrm{kt} \\
2660000 \mathrm{k} €\end{array}$ & $\begin{array}{l}1400 \mathrm{kt} \\
2660000 \mathrm{k} €\end{array}$ & $\times 31$ \\
\hline & Épichlorhydrine & & $10 \mathrm{kt}(0,9 \%)$ & $\begin{array}{l}300 \mathrm{kt}(23 \%) \\
450000 \mathrm{k} €\end{array}$ & $\begin{array}{l}600 \mathrm{kt}(60 \%) \\
900000 \mathrm{k} €\end{array}$ & $\times 60$ \\
\hline & Acide acrylique & & Quantités pilotes & $\begin{array}{l}450 \mathrm{kt}(10 \%) \\
810000 \mathrm{k} €\end{array}$ & $\begin{array}{l}900 \mathrm{kt}(20 \%) \\
1620000 \mathrm{k} €\end{array}$ & $\times \infty$ \\
\hline & Isoprène & & Quantités pilotes & $\begin{array}{l}50 \mathrm{kt} \\
11000 \mathrm{k} €\end{array}$ & $\begin{array}{l}100 \mathrm{kt} \\
220000 \mathrm{k} €\end{array}$ & $\times \infty$ \\
\hline & Éthanol & & $\begin{array}{l}50 \mathrm{kt} \\
35000 \mathrm{k} €\end{array}$ & $\begin{array}{l}430 \mathrm{kt} \\
301000 \mathrm{k} €\end{array}$ & $\begin{array}{l}800 \mathrm{kt} \\
560000 \mathrm{k} €\end{array}$ & $\times 16$ \\
\hline & Acide lactique & & $\begin{array}{l}420 \mathrm{kt} \\
966000 \mathrm{k} €\end{array}$ & $\begin{array}{l}420 \mathrm{kt} \\
966000 \mathrm{k} €\end{array}$ & $\begin{array}{l}900 \mathrm{kt} \\
2070000 \mathrm{k} €\end{array}$ & $\times 2$ \\
\hline & Sorbitol & & $\begin{array}{l}140 \mathrm{kt} \\
140000 \mathrm{k} €\end{array}$ & $\begin{array}{l}300 \mathrm{kt} \\
690000 \mathrm{k} €\end{array}$ & $\begin{array}{l}400 \mathrm{kt} \\
920000 \mathrm{k} €\end{array}$ & $\times 2,8$ \\
\hline & TOTAL intermédiaires mondial & & $\begin{array}{l}667,5 \mathrm{kt} \\
1247750 \mathrm{k} €\end{array}$ & $\begin{array}{l}10150 \mathrm{kt} \\
8097000 \mathrm{k} €\end{array}$ & $\begin{array}{l}13250 \mathrm{kt} \\
13630000 \mathrm{k} €\end{array}$ & $\times 20$ \\
\hline USA & & 7983 kt (8 \%) & $10750 \mathrm{kt}$ & $16148 \mathrm{kt}$ & & $\times 2$ \\
\hline Europe & & 16,8 milliards $€$ & 8 milliards $€$ & 25 milliards $€$ & & \\
\hline
\end{tabular}

* Facteur multiplicatif ; les données sont indiquées en volume (ktonnes) et parts de marché (\%).

** Prédiction de McKinsey pour 2010, ce ne sont pas des chiffres avérés pour 2010.

*** Estimation prudente supposant que le meilleur scénario prédit par McKinsey pour 2010, i.e. 20 \% de la production de l'industrie chimique biosourcé, sera atteint en 2020.

tions « bulk » (1,3-propanediol, PLA. . .) avec de nombreuses usines implantées sur leur territoire. En Europe, les produits biosourcés font partie des six marchés porteurs identifiés par la Commission européenne (DG entreprise et industrie) dans le cadre du "Lead Market Initiative " (LMI), ou "Initiative marchés porteurs ». Le LMI a pour objectif d'identifier et de soutenir les secteurs d'activité totalement nouveaux, sur lesquels I'Union européenne peut développer un avantage concurrentiel. Un rapport concernant des mesures spécifiques pour promouvoir l'introduction sur le marché des produits biosourcés innovants a été publié en novembre 2009. En 2009, l'industrie chimique européenne utilisait 8 à $10 \%$ de matières premières renouvelables pour produire diverses substances chimiques, ce qui correspond à moins de $2 \%$ des surfaces agricoles cultivées. Des estimations projettent une superficie de $4 \%$ à I'horizon 2020. Par ailleurs, les associations Europabio et
ERRMA préconisent d'ajouter un objectif de $20 \%$ de produits biosourcés d'ici 2020 à la stratégie européenne "EU 2020 Strategy \& the EU Climate Change \& Energy Strategy ».

En France, l'industrie chimique française $s^{\prime}$ est engagée à passer d'une utilisation de $8 \%$ de matières premières $\mathrm{d}^{\prime}$ origine végétale en 2010 à $15 \%$ d'ici à 2017 et à utiliser tous les types de ressources agricoles et cellulosiques ainsi que les déchets et les co-produits. En ce qui concerne des données chiffrées pour la France, il n'existe à l'heure actuelle que celles issues de l'étude Alcimed réalisée en 2007 pour le compte de l'ADEME (tableau 2).

Une analyse fine des potentiels de ces marchés à court, moyen et long termes permet de dégager de grandes tendances :

- à court terme (horizon inférieur à 5 ans) : i) fort développement de la synthèse de produits biosourcés possédant une structure chimique identique à celle des produits d'origine pétrochimique (molécules identiques) à partir de procédés de transformation aval non modifiés; ii) exploitation des intermédiaires chimiques biosourcés qui arrivent déjà sur le marché (exemples : acide succinique, épichlorhydrine, isosorbide ... ) et d'autres issus de projets qui sont à l'étape de démonstrateurs ou pilotes industriels et qui devraient atteindre le stade commercial d'ici 5 ans (exemples : acroléine, acide acrylique...) ; iii) développement de produits biosourcés innovants utilisant les caractéristiques propres des végétaux disponibles actuellement suivant les domaines (exemples : polymères : Ingeo ${ }^{\mathrm{TM}}$, MaterBi $^{\mathrm{R}}{ }$, Gaïalene ${ }^{\mathrm{R}}$, Bioplast ${ }^{\mathbb{R}}$, Rilsan ${ }^{\mathbb{R}}$, Pebax ${ }^{\mathbb{R}} \ldots$; composites ; lubrifiants : DIESTERub ${ }^{\mathbb{R}} \ldots$...); - à moyen terme (horizon 2020), des produits biosourcés obtenus à partir de ressources lignocellulosiques et de déchets devraient faire leur apparition sur le marché. En effet, de nombreux projets de recherche industrielle et démonstrateurs de recherche sont en 
Tableau 2. Marchés des produits biosourcés issus de matière premiere locale en Europe actuellement et prospectives pour 2020)

\begin{tabular}{|c|c|c|c|c|}
\hline & 2008-2010 & $\begin{array}{l}2020 \\
\text { sans contraintes } \\
\text { politiques }\end{array}$ & $\begin{array}{l}2020 \\
\text { avec contraintes } \\
\text { politiques }\end{array}$ & $\begin{array}{l}\text { Croissance avec } \\
\text { contraintes } 2020 \\
\text { vs } 2010^{*}\end{array}$ \\
\hline Intermédiaires chimiques & $\begin{array}{l}138,5 \mathrm{kt} \\
249550 \mathrm{k} €\end{array}$ & $\begin{array}{l}2030 \mathrm{kt} \\
1619400 \mathrm{k} €\end{array}$ & $\begin{array}{l}2650 \mathrm{kt} \\
2726000 \mathrm{k} €\end{array}$ & $\times 19$ \\
\hline \multicolumn{5}{|l|}{ Matériaux biosourcés } \\
\hline Polymères & $\begin{array}{l}260 \mathrm{kt} \\
1085000 \mathrm{k} €\end{array}$ & $769 \mathrm{kt}$ & $\begin{array}{l}2550 \mathrm{kt} \\
9980000 \mathrm{k} €\end{array}$ & $\times 10$ \\
\hline Matériaux composites & $\begin{array}{l}315 \mathrm{kt}(13 \%) \\
725000 \mathrm{k} €\end{array}$ & & $\begin{array}{l}830 \mathrm{kt}(28 \%) \\
1845000 \mathrm{k} €\end{array}$ & $\times 2,6$ \\
\hline \multicolumn{5}{|l|}{ Produits fonctionnalisés } \\
\hline Lubrifiants & $\begin{array}{l}137 \mathrm{kt}(3 \%) \\
411000 \mathrm{k} €\end{array}$ & $277 \mathrm{kt}$ & $\begin{array}{l}420 \mathrm{kt} \\
1260000 \mathrm{k} €\end{array}$ & $\times 3$ \\
\hline Solvants & $1,5 \%$ & $12 \%$ & $40 \%$ & $\times 26$ \\
\hline TOTAL & $\begin{array}{l}8-10 \%(14) \\
850,5 \mathrm{kt}\end{array}$ & & 16 milliards $€$ & \\
\hline
\end{tabular}

* Facteur multiplicatif ; les données sont indiquées en volume (ktonnes) et parts de marché (\%).

cours dans l'optique du développement des biocarburants avancés, ce qui devrait permettre d'accéder à des molécules plateformes nécessaires à la production industrielle de produits biosourcés ;

- à long terme (horizon 2030), on peut envisager que des produits innovants utilisant les caractéristiques propres des végétaux auront fait leur apparition sur le marché. En complément des procédés biosourcés à base de lignocellulose, ceux à partir de micro-algues devraient avoir été développés, aidés par les projets de recherche industrielle des biocarburants d'origine algale.

\section{Les freins}

Aujourd'hui, bien que de nombreuses initiatives soient prises en France comme dans I'Union européenne pour favoriser la pénétration des produits biosourcés sur le marché, il subsiste encore d'importants freins. Ces freins portent notamment sur : i) les prix des produits biosourcés qui sont souvent plus chers que ceux de leurs équivalents $\mathrm{d}^{\prime}$ origine fossile à fonctionnalité identique; ii) la connaissance insuffisante des attentes sociétales des industriels afin de mieux cibler les segments d'applications où le consommateur acceptera un surcoût ou acceptera le produit proposé ; iii) l'absence de réglementations adaptées aux produits biosourcées : les réglementations actuelles sont toutes mises en place pour les produits pétrochimiques et ne prennent pas en compte les spécificités des produits biosourcés. Les labels officiels (Ecolabel européen, NF environnement, etc.) ou privés existants sont inadaptés, car ils reposent sur des référentiels de certification dont les critères ne sont pas réellement pertinents pour les produits biosourcés; iv) la réglementation REACH, bien que potentiellement favorable pour les molécules issues de la biomasse, peut avoir un coût pour les produits biosourcés surtout lorsqu'ils sont concurrencés par des produits homologues importés de pays hors $\mathrm{d}^{\prime}$ Europe non soumis à une réglementation de ce type.

\section{Conclusion}

En conclusion, la biomasse représente I'une des alternatives principales à l'utilisation des ressources fossiles pour l'industrie chimique en tant que matière première carbonée. La France dispose de ressources agricoles et forestières abondantes pour assurer le développement des produits biosourcés sans entrer en concurrence avec les usages alimentaires et énergétiques. La filière chimie du végétal se structure en France grâce à la mise en place de structures fédératrices qui rassemblent les acteurs de l'ensemble de la chaîne de valeur, depuis la production de la biomasse, la distribution du produit fini, en passant par toutes les étapes intermédiaires de transformation réalisées par les agro-industriels, les chimistes, les biotechnologues, les plasturgistes... Cette filière a besoin d'investissement pour financer l'innovation industrielle, anticiper la disparition inéluctable des ressources pétrolières, la hausse progressive et persistante du prix du baril et aussi pour mieux gérer les ressources végétales en termes de disponibilité, d'approvisionnement, d'accessibilité, de durabilité, de prix des ressources. .. Aujourd'hui, de plus en plus de spécialistes s'accordent pour dire que le développement de la filière de la chimie du végétal, intégrée au sein de la chimie durable, pourrait contribuer à une réindustrialisation partielle de la France et ainsi stimuler la création de nombreux emplois non délocalisables dans l'ensemble des filières dans lesquelles elle intervient : bioraffinerie, agriculture, environnement (gestion des déchets). En attendant, pour accompagner la diffusion des produits biosourcés actuels et en développement, un soutien des pouvoirs publics est indispensable. II se traduirait par des mesures fiscales avantageuses (par exemple, crédit d'impôt accordé sur la base du contenu végétal de certains intermédiaires ou produits finis, TVA réduite...), l'introduction d'une préférence aux produits biosourcés dans les achats publics pour une liste 
déterminée de produits, la mise en place $d^{\prime}$ une labellisation permettant de valoriser les bénéfices environnementaux des produits biosourcés.

\section{POUR EN SAVOIR PLUS}

La chimie en France en bref. UIC édition, 2008.

Groupe de réflexion stratégique. Avenir de I'industrie chimique en France à l'horizon 2015. Mai 2005.

PIPAME. Mutations économiques dans le domaine de la chimie. Février 2010.

ADEME. Feuille de route R\&D de la filière chimie du végétal. ADEME, avril 2010.

Suschem, Feuille de route. France 2010.

ADEME. Panorama et potentiel de développement des bioraffineries. ADEME, octobre 2010.

Forum Economique Mondial. The future of industrial bioraffineries Rapport du Forum Economique Mondial, 2010.
USDA Procurement - Special procurement programs - Biopreferred. USDA Departmental Management, 2007.

USDA Procurement - Procurement Business opportunities/types of products and Services Purchased. USDA Departmental Management, 2005.

Taking bio-based from promise to market: measures to promote the market introduction of innovative biobased products. A report from Ad-hoc Advisory Group for biobased products in the framework of the European Commission's Lead Market Initiative. European Commission Entreprise and Industry. Published 3 November, 2009.

ADEME. Étude d'opportunité sur développement de la chimie du végétal. Note interne ADEME, janvier 2011. Non publiée.

Building the Bio-Economy by 2020 . Flagship initiative within the Europe 2020 strategy.

France Biotech, juin 2010.

Rapport de la Royal Academy of engineering "Synthetic Biology, Scope, application and implications", May 2009.
CGDD. Étude "filières vertes " du Commissariat General au Développement Durable. CGDD. 2009.

Boston Consulting Group. Étude indépendante d'évaluation du Grenelle de l'environnement du Boston Consulting Group. "Réflexions sur le portefeuille de mesures Grenelle de l'environnement, 2009.

ADEME, Alcimed. Marché actuel et prospectif des bioproduits industriels et des biocarburants en France. ADEME, Alcimed, 2007.

Compilation de données issues de diverses sources par I'ACDV et ERRMA, 2010.

ADEME, Bio Intelligence Service. Évaluation des besoins en labellisation et étiquetage des produits incorporant des matières d'origine renouvelable et comparaison des méthodes existantes. Promotion des bioproduits et biomatériaux. ADEME, Bio Intelligence Service, décembre, 2007.

ADEME, Bio Intelligence Service. Étude d'une méthodologie simplifiée pour la réalisation des ACV des bioproduits. Rapport final ADEME, Bio Intelligence Service, 2009. 